\title{
Impact of Allocation the Resources on Decision-Making in Course of Planning Processes through Design Predictive Model Using Fuzzy Logic Approach
}

\author{
Maged Mourad Said Mekhail \\ Faculty of Engineering, Ain Shams University, Cairo, Egypt \\ Email: nmnago@aol.com
}

How to cite this paper: Mekhail, M.M.S. (2017) Impact of Allocation the Resources on Decision-Making in Course of Planning Processes through Design Predictive Model Using Fuzzy Logic Approach. Journal of Building Construction and Planning Research, 5, 146-158.

https://doi.org/10.4236/jbcpr.2017.54011

Received: October 7, 2017

Accepted: November 14, 2017

Published: November 17, 2017

Copyright $\odot 2017$ by author and Scientific Research Publishing Inc. This work is licensed under the Creative Commons Attribution International License (CC BY 4.0).

http://creativecommons.org/licenses/by/4.0/

\begin{abstract}
One of the most important elements of the performance in planning, at the field of planning engineering, is to identify the resources and then distribute the resources on activities, and before establishing the time schedule for activities. The planning in project management, was not merely limited to the making schedule for the range of activities, or the development of the relations for those activities through Microsoft or Primavera (P6), so it can be through two identical projects for the same logical sequence of the activities network to both of them and have the same start time with identical activities and both in same location. But both the ends of the actual time will be different to both of them. The reason for this is back to the difference at the quality of the planning performance between each of the two projects. Accordingly, that paper designed a model to estimate the perform of tools from field data using conclusion fuzzy system to determine the impact of higher produce between this supply in light of the location and conditions of work.
\end{abstract}

\section{Keywords}

Planning Engineering, Fuzz Logic Approach, Modelling,

Decision-Making, Allocation Resources, Simulation Modeling

\section{Introduction}

A number of studies and researches about construction projects management have focused predominantly on problem control of time and cost saving by using many of the traditional cognitive methods. 
One of those methods is control of the supplies' distribution on activities while maintaining the important role played by the planning network in the management of supplies, it proposes a method for the division of supplies based upon the time criterion. This method depends on a matrix expressing short times [1]. Other studies try to find out the ideal critical path for projects networks, with design and application of genetic algorithms to find out a critical path in an attempt to achieve the many objectives and determine the real time for project's execution [2]. Another research worked on simulating construction operations with the application of dynamic simulation system in order to accurately obtain the cycle time of the process [3]. But some studies have not neglected to study the effect of subjective variables on construction process [4].

\section{Problem Statement}

Many of these studies have neglected the effect of determining the supplies selection. There are plentiful supplies that can be used within the same work, certainly, there will be a different effect for each of them to determine the age of activity, and uncertainty is an entrenched characteristic of most construction projects. Hence, the problem arises.

Bauer et al. [5] was able to prove that the fuzzy logic was a good way to reach a specific result of knowing information, or blurry (fuzzy data).

In contrast to traditional logic that requires a deep understanding of the problem and the solution of accurate algorithms of the data, fuzzy logic embodies thinking by the other way, which allows model complex data systems and finds their solution, despite that this type of data in their entirety consists of systems and that it can change from static or continuous form, which is usually hard to apply in the conventional mathematics formats [6].

This occurs through the use of the thoughts and the accumulated information and experience. Figure 1 shows the fuzzy logic steps configuration that is according to numerous studies such as the study [7] [8].

So through this study, we can make full use of performance data rates for construction in drawing conclusions that are compatible with the self-variables in the construction site and that have usually been difficult to achieve good results according to these variables. Therefore the design of the fuzzy model approach facilitates the extraction of these results depending on the data rates that are extracted from analysis base standard.

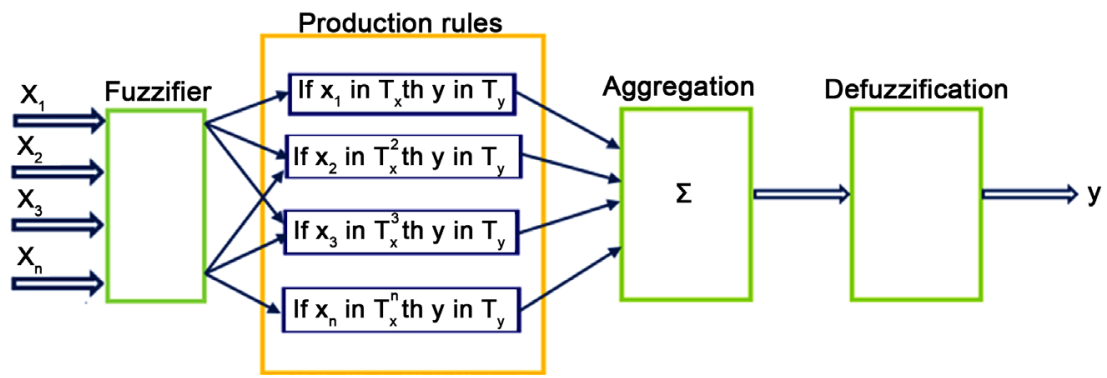

Figure 1. Schematic diagram of a fuzzy inference system. 


\section{Scope}

In burrowing event log and when known work registration, we find that more than supples of tools registration can be used for complete the work, and at the same time, we should make the decision to choose the best from those tools to complete the work. Particularly, those tools are the most efficient, and could also lead to the production of many work types.

Now, it is necessary for the planning engineer to find a technique to ease him to make the best decision in choosing the right supply. This can be achieved through finding a way to help us predict tool rendering, and also provide work epoch in order to complete the project. This is, in addition to our dependence on both the number and type of supply tools, supposed to do the task. Moreover, the circumstances could facilitate or hinder the completion of the work due to the choice of tools supply.

The aim of the designed model is to estimate the performance of tools from field data using conclusion fuzzy system and to determine the impact of higher performance between this tool in light of the location and conditions of work and then to choose from the most appropriate one.

\section{Modeling Resources Conditions}

In order to reach this solution, we need to use the supplies to construct activities [8], which contain three major elements: First, perform data rates for each resource; Secondly, Model Analytical to such data; Finally, model fuzzy sets multi-system in accordance with the foregoing, gathering data rates of yield to every supplier for every work equally. Then, convert these data that has been gathered to an analytical model, which is used to classify and analyze the data that is collected to obtain effectiveness of other supplies performance within each work.

Taking into consideration the factors affecting the performance of supplies, these recourses are one of the items that help the accuracy of a result of the selection. The impact of supplies on the project can be divided into two parts: direct and indirect.

Direct effect: it means the total or partial disruption of business due to machinery breakdown, obstruction of work, or lack of employees.

Indirect effect: it means the decrease of the work performance due to the inefficiency of the tools, lack of maintenance, inefficiency of labors, or the atmosphere circumstance in the location project [9].

\section{Perform Data}

It is a standard performance data crisp number for each supply-in this tool study-collection rates according to each activity, and often passes such rates depending on the performance rate of each company.

\section{Analytical Model Data Performance Rates}

A - Collecting performance rates data of the supplies. 
B-The classification and analysis of supplies according to type project and nature of the work environment.

C-The classification and analysis of shared supplies in the same activities, according to its production capacity.

D-Dividing data performance rates of supplies in each activity to groups.

E-From a data set should be created for the relation between quantitative and qualitative.

\section{A Fuzzy Model Design for Prediction the Quantitative to Determine the Equipment}

Notwithstanding the possible existence of several supplies be shared with each other in terms of the nature of work within the same activity, but each has a different effect is different in terms of the productivity of the work required, Leading to create a difference between the deadline and accomplish of work between each supplier and last [10].

On this basis, we can use fuzzy logic to find a relationship between the type of shared supplies in the same nature of activity work [11], and the amount of output for each of them, what makes it a proportional on impact to the deadline for completion of work Activity.

The following content discuss our model in detail we introduce some basic concepts related to intuitionistic fuzzy sets (IFS) and Interval-valued intuitionistic fuzzy sets [12]:

Definition 1. Let $X$ be a universe of discourse, then a fuzzy set is defined as:

$$
A=\{[x, \mu A(X)] \in x(X)\}
$$

which is characterized by a membership function

$$
\mu A: X \rightarrow[0,1]
$$

where $\mu A(x)$

$$
f(x ; a, b, c)=\{0 \text { for } x<a\},\{1 \text { for } x=b\}
$$

Definition 2. An IFS $A$ in $X$ is given by

$$
A=\{[x, \mu A(X), V a(x)] x \in(X)\}
$$

where $\mu A: X \rightarrow[0,1]$ and $v A: X \rightarrow[0,1]$, with the condition

$$
0 \leq \mu a(x)+V a(x) \leq 1, \forall x \in X
$$

The numbers $\mu A(x)$ and $v A(x)$ represent, respectively, the membership degree and nonmember ship degree of the element $x$ to the set $A$

Definition 3. For each IFS $A$ in $X$, if:

$$
\pi A(x)=1-\mu a(x)-v a(x), \forall x \in X
$$

Then $\pi A(x)$ is called the degree of indeterminacy of $x$ to $A$.

A fuzzy subset $A$ with an element $x$ has a membership function of $\mu_{A(x)}$. When the universe discourse $X$ is discrete and finite, this mapping can be expressed as 


$$
A=\frac{\mu_{A\left(x_{1}\right)}}{x_{1}}+\frac{\mu_{A\left(x_{2}\right)}}{x_{1}}+\cdots=\sum_{i} \frac{\mu_{A\left(x_{i}\right)}}{x_{i}}
$$

when the universe $X$ is continuous and infinite, the fuzzy set $A$ can be repented as:

$$
A=\int \frac{\mu_{A(x)}}{x_{1}}
$$

In this paper inference fuzzy system has been used through the emerging information and experience. And configuration steps fuzzy logic are shown in Figure 1 [13].

\section{A Numerical Example}

In this section, we present a numerical example to illustrate proposed method presented in this paper and show that the method is an effective method for determining supplies selection from multiple productivities [14].

We first make the following assumptions:

1) Determining supplies selection should be in bowering event.

2) In the type case normal soil dry flat, where yield varies rate and nature of the Tool, according to the Work type.

3) Which rate performance of supply in this soil type according to the data available will be to the: Track Bulldozer, track loader, wheeled loader.

4) The mission of this paper is to propose a method of decision-making to select of them is best in activity performance.

5) The productivity of each the Tool is inversely proportional to the displacement distance for each of them.

6) Displacement distance for each our Tool in the site of implementation is not a requirement to be identical with the rates of performance data which given.

7) Because of this, productivity in the site of implementation is unknown according to the data in performance rates.

Case 1: the value of the decision for example of the normal dry soil at the track bulldozer

Table 1 collected data (crisp value) for performance at horsepower \& distance of track bulldozer.

\begin{tabular}{|c|c|c|c|c|}
\hline \multicolumn{4}{|c|}{ Performance at distance/ $d$} & \multirow{2}{*}{ Horsepower } \\
\hline $30 \mathrm{~m}$ & $60 \mathrm{~m}$ & $90 \mathrm{~m}$ & $120 \mathrm{~m}$ & \\
\hline 450 & 205 & 100 & 70 & $75(D 4)$ \\
\hline 680 & 315 & 180 & 135 & $105(D 5)$ \\
\hline 840 & 450 & 275 & 190 & $140(D 6)$ \\
\hline 1270 & 580 & 450 & 315 & $180(D 7)$ \\
\hline 1810 & 995 & 680 & 520 & $270(D 8)$ \\
\hline 2490 & 1445 & 1015 & 795 & $385(D 9)$ \\
\hline
\end{tabular}

Table 1. Data collection. 


\section{Fuzzy Relation}

In this section, we illustrate the relation between the fuzzy input and the required out-put. This section describes the fuzzy inference process and uses the example of the two-input, one-output, Fuzzy inference is the process of formulating the mapping from a given input to an output using fuzzy logic [14].

Figure 2 shows the structure of the proposed solution consisting of inputs, reasoning rules and outputs is performance, two inputs they displacement distance and powerhouse as described.

Related to configuration in Figure 1, the configuration Simulink mat lab toolbox steps fuzzy logic are shown in the Figure 2.

The two inputs are denoted as:

$\mu_{A}(\mathrm{D})$ where

$$
\mathrm{D}=\{\mathrm{ST}, \mathrm{MD}, \mathrm{F}, \mathrm{TF}\}
$$

$\mu_{B}(\mathrm{PH})$ where

$$
\mathrm{PH}=\{\mathrm{VF}, \mathrm{FB}, \mathrm{MD}, \mathrm{SG}, \mathrm{SR}, \mathrm{PF}\}
$$

\section{Membership Function}

Table 2 is specified for two input and one output performance; few of them are presented as below.

Figure 3 provides the fuzzification of distance on Simulink related to Table 3 for input one.

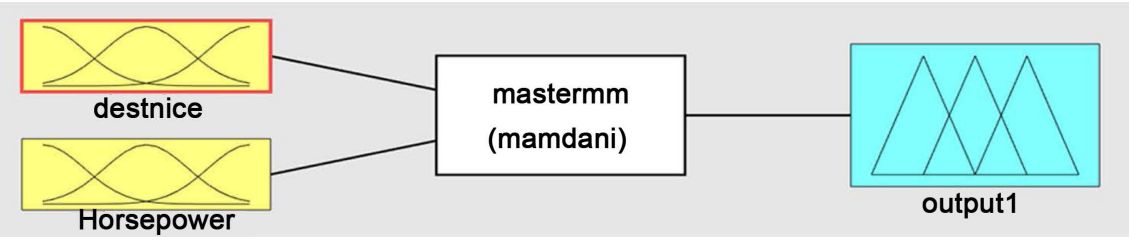

Figure 2. Fuzzy inference system.

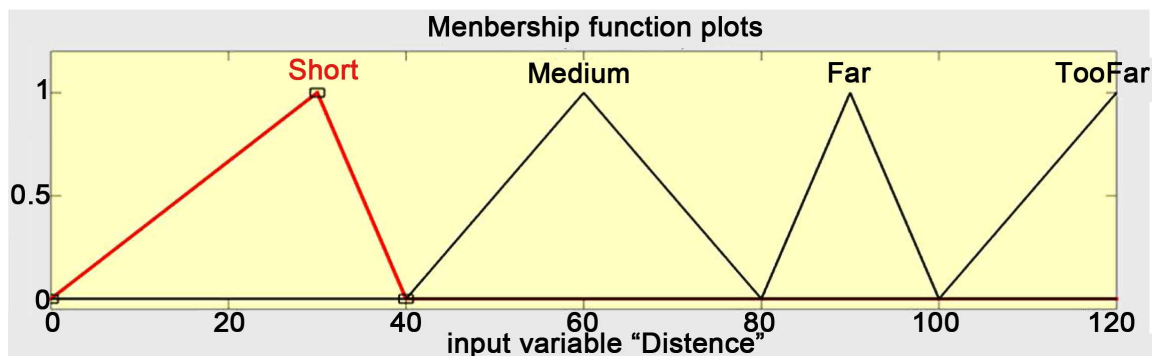

Figure 3. Fuzzy member ship function of distance-input (1).

Table 2. Input one of distance fuzzification.

\begin{tabular}{cc}
\hline Distance fuzzification & linguistic qualifier \\
\hline $0,30,40$ & short \\
$40,60,80$ & Medium \\
$80,90,100$ & Far \\
$100,120,160$ & Too far \\
\hline
\end{tabular}


Table 3. Input tow of H.P fuzzification.

\begin{tabular}{cc}
\hline H.P fuzzification & linguistic qualifier \\
\hline $0,75,77$ & Very feeble \\
$66,105,154$ & feeble \\
$77,140,231$ & Medium \\
$154,180,308$ & Strong \\
$231,270,385$ & Stronger \\
$308,385,462$ & powerful \\
\hline
\end{tabular}

Distance Fuzzification for input one, by Taking input and determine the degree to which belong to each set of the appropriate fuzzy sets via membership functions.

Figure 4 provides the fuzzification of H.P on Simulink, related to Table 4 for input $\mathrm{n}$. tow.

Table 4 provides Membership function of performance, the inference rules relate these input to the output perform consisting of six membership functions i.e. as: VERY LOW (VL), LOW (LW), MEDIUM (MD), HIGH (HG), VERY HIGH (VH), and EXCELLENT (EX).

In this paper, we use Mamdani type Fuzzy Inference Rules. As noted by [5], every rule represents a fuzzy relation. In our case, every rule represents the relation between the two inputs to an output.

Figure 5 shows, the complete membership Functions of performance which is denoted as:

$\mu \mathrm{O}$ (performance) where $\mathrm{O}=\{\mathrm{VL}, \mathrm{LW}, \mathrm{MD}, \mathrm{HG} \mathrm{VH}, \mathrm{EX}\}$

\section{Rule Base}

A fuzzy rule base contains a set of fuzzy rules $R$. A single if-then rule assumes the form "if $x$ is $T x$ then $y$ is Ty." An example of a rule might be "if service is high and experience is high, then salary is very high." For a multiinput, multioutput system,

$$
R=(R 1, R 2, \cdots, R n)
$$

$R i$ if $\left(x_{1}\right.$ is $T x_{1}, \cdots, x p$ is $\left.T x p\right)$ then

$$
(y 1 \text { in } T y 1, \cdots, y 4 \text { in } T y 4)
$$

Let us denote by $R$ the fuzzy relation between $\mathrm{D}, \mathrm{PH}$, and performance. As denoted in Teodorovic and Pavkoviv (1996), membership function $\mu R(x, y)$ of this fuzzy relation equals:

$$
\mu R(x, y)=\min \{\mu A(x), \mu B(y)\} \forall x, y
$$

In this paper, we denote the fuzzy relation as:

$$
\mu R(x, y)=\min \{\min (\mu \mathrm{A}(\mathrm{D}), \mu \mathrm{B}(\mathrm{HP})), \mathrm{PO}(\text { productivity })\} \forall x, y
$$




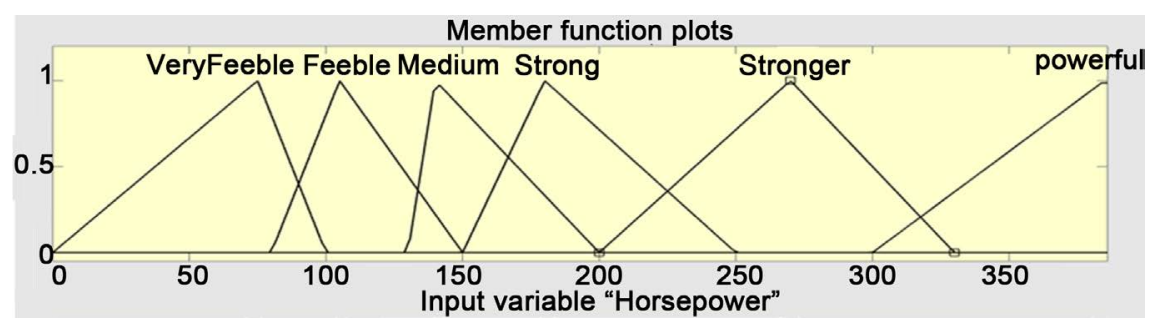

Figure 4. Fuzzy member ship function of P.H-input (2).

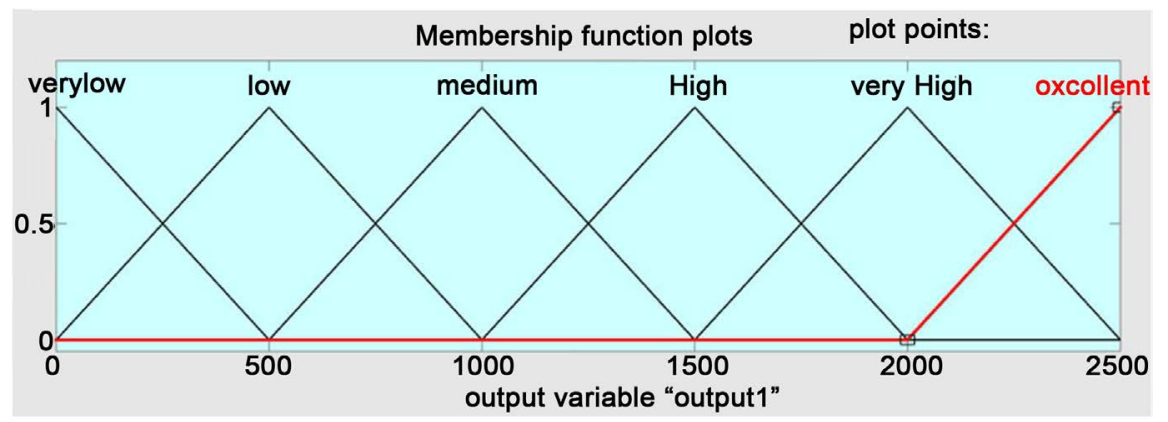

Figure 5. Fuzzy member ship function of Tool performance-output.

Table 4. Membership function of output performance.

\begin{tabular}{cc}
\hline P.fuzzification & Linguistic qualifier \\
\hline $0,450,500$ & Very low (1) \\
$450,680,1000$ & Low (2) \\
$680,840,1500$ & Medium (3) \\
$1000,1270,2000$ & High (4) \\
$1500,1810,2500$ & Very high (5) \\
$2000,2490,3000$ & Excellent (6) \\
\hline
\end{tabular}

where $x$ is input and $y$ is output.

$R 1$ : if distance ST and horsepower VF then performance is VL

$R 2$ : if distance $\mathrm{ST}$ and horsepower $\mathrm{FB}$ then performance is $\mathrm{LW}$

$R 3$ : if distance $\mathrm{ST}$ and horsepower $\mathrm{MD}$ then performance is $\mathrm{MD}$

R4: if distance ST and horsepower SG then performance is $\mathrm{HG}$

R5: if distance ST and horsepower SR then performance is $\mathrm{VH}$

R6: if distance ST and horsepower EX then performance is EX

$R i$ if $X_{1}$ is $T_{x} i$ and $X_{2}$ is $T_{2}$ then $y$ is $T y$

The fuzzy relations of those rules are shown as follow:

$$
\begin{aligned}
& \mu R(x, y)=\min \{\min (\mu \mathrm{ST}(\mathrm{D}), \mu \mathrm{VF}(\mathrm{HP})), \mu \mathrm{VL}(\operatorname{prod})\} \\
& \mu R(x, y)=\min \{\min (\mu \mathrm{ST}(\mathrm{D}), \mu \mathrm{FB}(\mathrm{HP})), \mu \mathrm{LW}(\operatorname{prod})\} \\
& \mu R(x, y)=\min \{\min (\mu \mathrm{ST}(\mathrm{D}), \mu \mathrm{MD}(\mathrm{HP})), \mu \mathrm{M}(\operatorname{prod})\}
\end{aligned}
$$


Figure 6 provides the Mamdani type Fuzzy Inference Rules. In this paper [16] as will work on the production of the output as.

\section{Aggregation}

Aggregation is a process whereby the outputs of each rule are unified. Aggregation occurs only once for each output variable. The input to the aggregation process is truncated output fuzzy sets returned by the implication process for each rule [18]. The out-put of the aggregation process is the combined output fuzzy set.

Table 5: The Fuzzy associative matrix: Therefore, this category can be facilitated through the collection of these fuzzy laws in the table, and the expression of each of the six rating categories for output number upward until reaching the highest rating (1:very low, $\cdots, 6$ : excellent) according to the following:

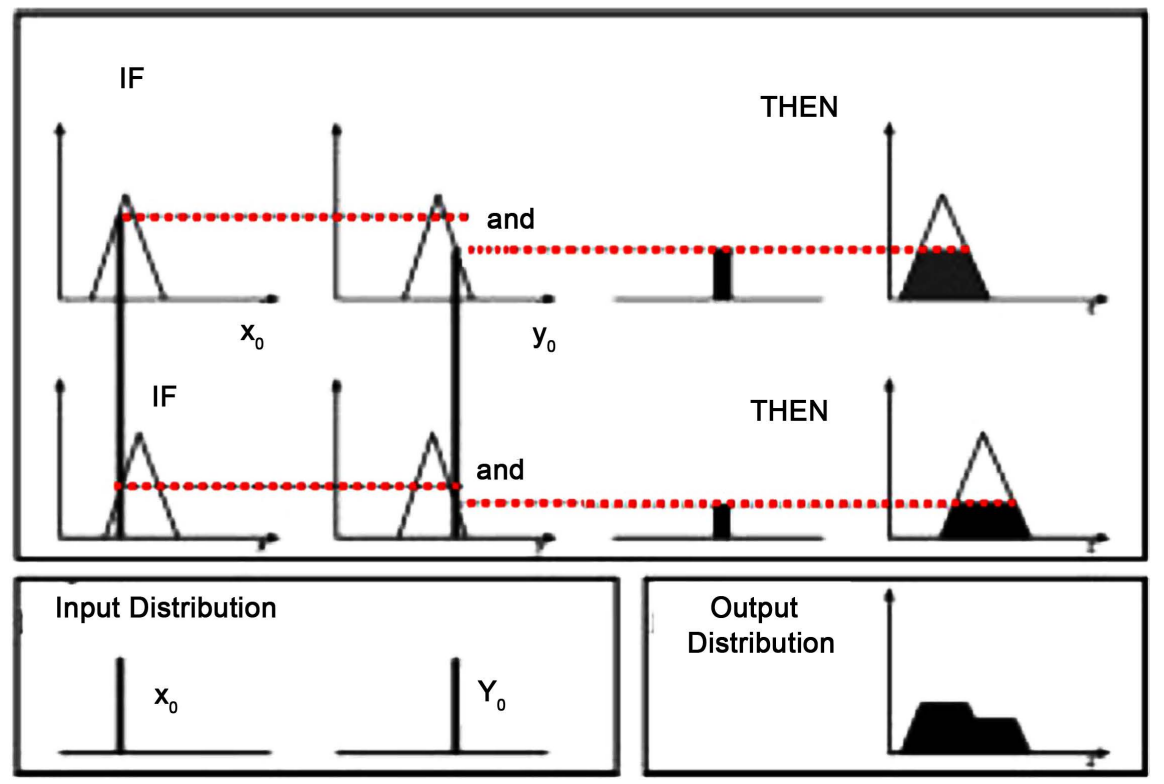

Figure 6. Mamdani inference system [17].

Table 5. The Fuzzy associative matrix.

\begin{tabular}{ccccc}
\hline & \multicolumn{4}{c}{ Performance at distance /D } \\
H.P & ST & MD & F & TF \\
\hline VF & V L & V L & V L & V L \\
FB & LW & V L & V L & V L \\
MD & MD & V L & V L & V L \\
SG & HG & LW & V L & V L \\
SR & VH & MD & LW & LW \\
PF & EX & HG & MD & MD
\end{tabular}


In the previous phase, has been put each of four categories: distance of the supplies and six categories for the Tool's horsepower, and therefore, the maximum number of possible cases of 24 cases, and therefore the system needs to a maximum of 24 fuzzy law for case n. one, at track bulldozer, but some of these laws can sometimes shortcut, it is possible shortcut six laws by ignoring the supply's ability as an example.

"If the distance is too far then the yield is very low"

Where the Tool's ability to ignore given the opportunity to shorten the six specifications (combination) and combine all description of the six descriptions of the ability of the supply and displacement distance.

Figure 7 provides the result of an Aggregate according to the Mamdani type Fuzzy Inference Rules.

\section{3D Surface Viewer for FIS}

Figure 8 provides the Surface Viewer is use for presenting the mapping from two inputs to one output. This capability allows keeping the calculation time reasonable for complex problems three dimension view as.

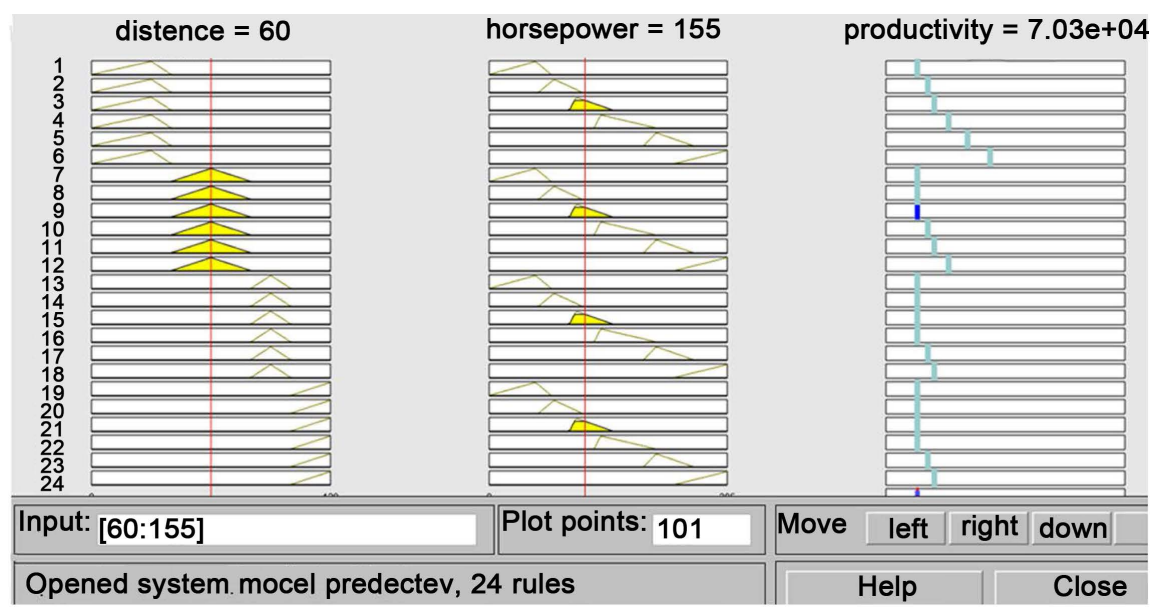

Figure 7. Roles viewer [15].

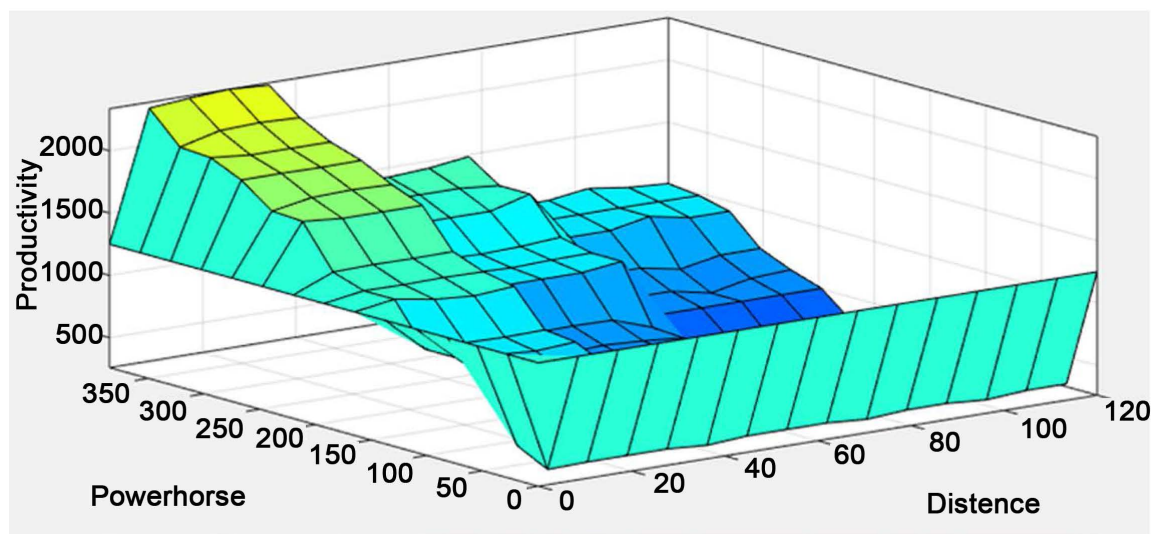

Figure 8. Surface viewer. 


\section{Defuzzification Methods}

The most common defuzzification methods are presented and compared in this paper i.e. Centre of Area.

\section{Center of Area/Gravity}

It is one of the most commonly used defuzzification Techniques. This method determines the center of the area of the combined membership functions [4]. [6] calculated the centroid or center of gravity (COG) of The area under the membership function [19]. In this method, the defuzzifier determines the center of area (centroid) of B what is meant here of space is space of decision, along with the lines of the center of gravity, and uses that value as the output of the FLS [20]. For A continuous aggregated fuzzy set, the centroid is given by:

$$
F_{C O G}^{1}(\tilde{A})=\frac{\int_{x} \mu \tilde{A}(x) \cdot x \mathrm{~d} x}{\int_{x} \mu \tilde{A}(x) \mathrm{d} x}
$$

where $F^{1}$ the defuzzified output and $\mu A(x)$ is the output fuzzy set after aggregation of individual implication results.

Another formula

$$
Y 0=\frac{\int y 1 Y(y 1)+y 2 Y(y 2)}{\int Y(y 1)+Y(y 2)}
$$

when

$$
\begin{gathered}
Y=\text { Fuzzy set to which it belongs decision } \\
Y_{0}=\text { final decision, } \mu=\text { the degree of organic } \\
y_{1}=\text { the first decision, } y_{2}=\text { the second decision }
\end{gathered}
$$

\section{Model Application Method}

After we finished the design Tools unit system, we can through this model Predictive to the perform of the Tools unit, at soil type, in this case, is normal dry, at any transport distance to be determined according to the layout of the general site, and at any horsepower which determines according to the project budget, within the limits of the data that have been entered into this system.

In this case, it will make it easier for decision makers to identify selection type the supply which should work out through the largest value, Identified in accordance with the highest performance according to the circumstances of the Site, and the highest Tool in perform among the rest of the supply is selected.

\section{Conclusions}

The process of allocating supplies is one of the most important planning processes, and assists in completing the project and not only just contributes to determining activities durations in order to prepare project schedule, but also has extended to impact what is far beyond the project scope, and this is what we need in all activities.

As a result, the planning engineer is considered as the designer for the pro- 
cesses performance on the site before starting the project implementation, which is called planning processes. Thus, the planner is not less important than any designer of the project, whether architect designer or construction designer. $\mathrm{He}$ classifies the supplies that will be used to perform the project. It is done in the same pattern by the consultant to obtain the required quality in the short time within the budget limit of the project.

Therefore in all cases, the planning engineer needs a way with a clear methodology to help him to make a decision during designing to implement the project before starting the work.

Then, it would be easier to monitor and follow up the work. Accordingly that, the importance of decision making is evident in accordance with this study, and this aim can be achieved easily, we should answer the following questions: what is the goal of the work? What should be done to reach the desired result? What are reasons for deviation?

The answer to these questions is to make the decision-making be in accordance with what has already been explained. In reality, this has been done according to the achieved results and under performance rate data.

\section{References}

[1] Gonoud's Study (2005) Coordination of the Construction Projects Execution in Case Supplies Hare Been Allocated Based upon Matrixes Logarithm. Ph.D. Thesis, Aleppo University, Syria.

[2] Talae's Study Entitled (2011) A Plan of Genetic Algorithms to Find Out the Ideal Critical Path for Projects Works Network. Unpublished Study, Faculty of Computer Since and Mathematics, Aleppo University, Syria.

[3] Akhavian, R. (2011) Dynamic Simulation of Construction Activities Using Real Time Field Data Collection. 18th Annual Workshop of European Group for Intelligent Computing in Engineering EG-ICE, 1-10.

[4] Sweis, G.J. (2016) Factors Affecting Construction Labor Productivity. Journal of Construction Management, 16, 138-149.

[5] Teodorovic, D. and Pavkovic, G. (1996) The Fuzzy Set Theory Approach to the Vehicle Routing Problem When Demand at Nodes Is Uncertain. Fuzzy Sets and Systems, 82, 307-317. http://dx.doi.org/10.1016/0165-0114(95)00276-6

[6] Samarkandi, H.I. (1995) Dynamic System Modeling, Identification, and Control: A Fuzzy-Logic Based Approach. Unpublished M.Sc. Thesis, Dept. of Computer Engineering, College of Computer and Information Science, King Saud University, Riyadh.

[7] Gassoumi, H., Ellington, J.J., Nguyen, H.T. and Prasad, N.R. (2000) A Neuro-Fuzzy Approach for Insect Classification. The WAC2000, Maui, 11-16 June 2000.

[8] Thomas, H., Maloney, W., Horner, R., Smith, G., Handa, V. and Sanders, S. (1990) Modeling Construction Labor Productivity. Journal of Construction Engineering and Management, 116, 705-726. http://dx.doi.org/10.1061/(ASCE)0733-9364(1990)116:4(705)

[9] Chan, A.P.C., Chan, D.W.M. and Yeung, J.F.Y. (2009) Overview of the Applications of Fuzzy Techniques in Construction Management Research. Journal of Construction Engineering and Management, 135, 1241-1252. 
http://dx.doi.org/10.1061/(ASCE)CO.1943-7862.0000099

[10] Shang, K.L. and Hossen, Z. (2013) Applying Fuzzy Logic to Risk Assessment and Decision-Making. Casualty Actuarial Society, Canadian Institute of Actuaries, Society of Actuaries, Vol. 2, 209-218.

[11] Makulsawatudom, A., Emsely, M. and Sinthawanarong, K. (2004) Critical Factors Influencing Construction Productivity in Thailand. The Journal of KMITNB, 14, 1-6.

[12] Zadeh, L.A. (1965) Fuzzy Sets. International Journal of Control, 8, 338-353. http://dx.doi.org/10.1016/S0019-9958(65)90241-X

[13] López, J.A., Araque Campo, R. and Matiz Rubio, A. (2014) Automixer: Equipment for the Reduction of Risks Associated with Inadequate Oxygen Supply. The Journal of Ingeniería e Investigación, 34.

[14] Muqeem, S. (2011) Construction Labor Production Rates Modeling Using Artificial Neural Network. Journal of Information Technology.

[15] MathWorks (2015) Fuzzy Logic Toolbox User's Guide, for the Use of Matlab. The Math Works Inc..

[16] Mamdani, E. and Assilian, S. (1975) An Experiment in Linguistic Synthesis with a Fuzzy Logic Controller. International Journal of Man-Machine Studies, 7, 1-13. http://dx.doi.org/10.1016/S0020-7373(75)80002-2

[17] Computer Science Department at Princeton University, Archive Website. http://www.cs.princeton.edu/courses/archive/fall07/cos436/HIDDEN/Knapp/fuzzy0 04.htm

[18] Bianchini, A. (2007) Prediction of Pavement Performance through Neuro-Fuzzy Reasoning. New Mexico State University, Las Cruces.

[19] Rao, D.H. and Saraf, S.S. (1995) Study of Defuzzification Methods of Fuzzy Logic Controller for Speed Control of a DC Motor. IEEE Transactions, 782-787.

[20] Runkler, T.A. (1996) Extended Defuzzification Methods and Their Properties. IEEE Transactions, 694-700. http://dx.doi.org/10.1109/fuzzy.1996.551822 